\title{
Common Envelope Evolution: Implications for Post-AGB Stars and Planetary Nebulae
}

\author{
J. Nordhaus \\ National Technical Institute for the Deaf, Rochester Institute of Technology, \\ One Bausch and Lomb Dr., Rochester, NY 14623, USA \\ email: nordhaus@astro.rit.edu \\ Center for Computational Relativity and Gravitation, Rochester Institute of Technology, \\ One Bausch and Lomb Dr., Rochester, NY 14623, USA
}

\begin{abstract}
Common envelopes (CE) are of broad interest as they represent one method by which binaries with initially long-period orbits of a few years can be converted into short-period orbits of a few hours. Despite their importance, the brief lifetimes of CE phases make them difficult to directly observe. Nevertheless, CE interactions are potentially common, can produce a diverse array of nebular shapes, and can accommodate current post-AGB and planetary nebula outflow constraints. Here, I discuss ongoing theoretical and computational work on CEs and speculate on what lies ahead for determining accurate outcomes of this elusive phase of evolution.
\end{abstract}

Keywords. stars: AGB and post-AGB, (stars:) binaries (including multiple): close, stars: magnetic fields, stars: mass loss, (stars:) planetary systems, stars: evolution

\section{Introduction}

The extreme, non-spherical nebular morphologies observed in post-asymptotic-giantbranch (post-AGB) stars and their planetary nebulae (PNe) progeny are often attributed to interactions with orbiting companions. As low-mass stars $\left(\lesssim 8 M_{\odot}\right)$ evolve off the main sequence, expansion of the primary's radius is accompanied by strong mass loss, and affords new opportunities for powering outflows in binary systems. Potential shaping scenarios range from mild shocking of the outflow as the wind moves past the secondary to more extreme events such as Roche Lobe overflow and common envelope phases (Nordhaus et al. 2007, Chen et al. 2016).

The progenitor systems that are expected to incur such interactions are potentially common and span a range of binary types. For example, stellar, sub-stellar or compact objects initially orbiting $\lesssim 10$ AU during the main sequence are expected to plunge into their host stars during the giant branches (Nordhaus et al. 2010, Nordhaus \& Spiegel 2013). Rapid inspiral on fast timescales leads to a significant reduction in separation between the companion and the giant's core (Paczynski 1976). As the orbit shrinks, significant energy and angular momentum are transferred to the common envelope itself.

If the secondary is of sufficient mass, the $\mathrm{CE}$ can be ejected with the system emerging as a short-period binary (see Figure 1). If the companion is of insufficient mass to survive the interaction, it will be tidally disrupted as it approaches the giant's core (Nordhaus \& Blackman 2006). The material from the disrupted companion forms an accretion disk inside the CE that is cold and dense compared to the ambient gas (Nordhaus et al. 2011). Even in these cases, accretion onto the proto-white dwarf core can eject the envelope resulting in a "single" star that, in effect, has been strongly influenced by the presence of a companion. Therefore, one complication in observationally determining the fraction of PN systems that evolved from binary systems is that: 
a.

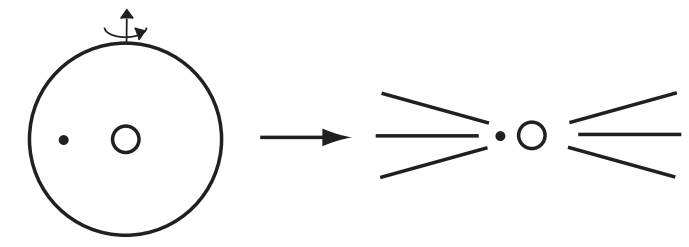

b.
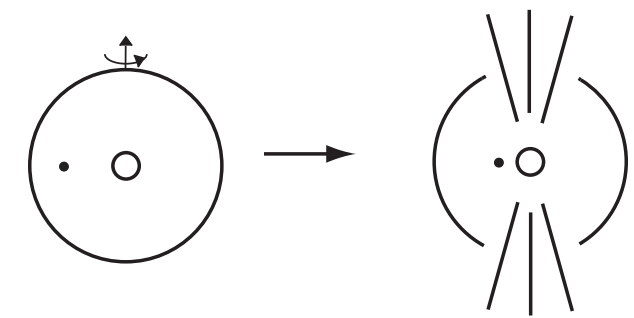

c.

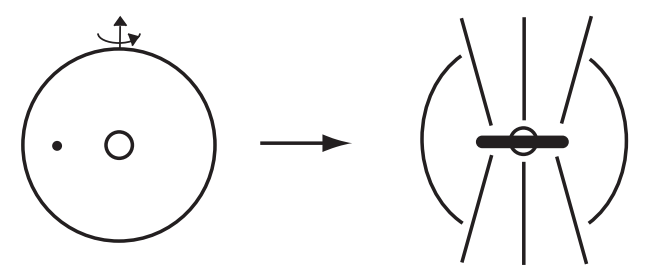

Figure 1. Possible outcomes of CE evolution. (a.) The companion directly ejects the envelope resulting, survives the CE and emerges as a post-CE binary (b.) Strong differential rotation in the $\mathrm{CE}$ induced by the plunging companion coupled with the presence of a deep convective zone, triggers a dynamo in the envelope resulting in a collimated bipolar outflow (c.) The companion is shredded into an accretion disk around the core which drives a collimated outflow and can lead to the formation of a highly-magnetized white dwarf (Nordhaus et al. 2011).

(i.) the absence of a detected companion does not imply that the progenitor system was a single star.

The converse is also highly applicable as common envelope phases are not the sole method for reducing orbital separation. In hierarchical three-body systems, Kozai-Lidov cycles exchange orbital inclination with eccentricity of the inner binary (Fabrycky \& Tremaine 2007). In essence, the eccentricity of the inner binary can be pumped near unity at which point the orbit can circularize due to tidal dissipation. As the tertiary is distant, such systems would appear as post-CE binaries. Additionally, multi-body systems where $n>3$ posses many methods that can generate close encounters. Therefore, we also have the added complication that:

(ii.) the detection of a short-period companion does not imply that the progenitor system evolved through a CE phase.

Since the white-dwarf mass function is strongly peaked, the population of short-period companions around low-mass white dwarfs may represent, at least in aggregate, a reasonable proxy for true descendants of CE phases. Disentangling the evolutionary outcome of a given system, let alone a population, requires a sufficient degree of fidelity from theoretical and numerical studies in conjunction with observational constraints. 


\section{Accretion during CEs satisfy observed post-AGB outflow constaints}

One area which has recently had success in constraining formation scenarios for postAGB systems comes from kinematic observations of large-scale outflows (Bujarrabal et al. $2001)$. From the measured outflow momenta, a minimum accretion rate, $\dot{M}_{\text {obs }}$, necessary to power the outflow can be derived (Blackman \& Lucchini 2014). The minimum accretion rates can be directly compared to theoretical models of engine-driving scenarios under the assumption of an accretor (main-sequence star or white dwarf) and the assumption that all accreted power goes into the outflow, $\dot{M}_{\text {max theory }}$. If $\dot{M}_{\text {max }}$, theory $<\dot{M}_{\text {obs }}$, that particular scenario can be confidently ruled out.

Recently, Blackman \& Lucchini (2014) used observations from a sample of 19 post-AGB systems with kinematic outflow measurements. The authors considered both white dwarf and main-sequence accretors and four accretion paradigms: Bondi-Hoyle-Littleton (BHL) wind accretion, Roche-Lobe overflow consistent with the rate for the Red Rectangle, Wind-Roche-lobe overflow, and accretion during a common envelope phase. BHL wind accretion and Wind-Roche-lobe overflow were ruled out as explanations for the vast majority of objects. Roche-lobe overflow at the rate measured in the Red Rectangle could plausibly accommodate $\sim 1 / 3$ of the sample. However, the only scenario that successfully powers all objects was accretion during a CE phase. This does not imply that common envelope accretion powers these systems, only that there is sufficient power available during the CE phase to explain the observed outflows.

\section{CEs with Planetary and M-Dwarf Companions}

Planets around main-sequence stars represent a reservoir of potential companions that might be expected to incur a CE phase and provide a source of accretion during the giant phases. Figure 2 shows the fates of known planetary systems with masses at least Jupiter's and host-star masses greater than or equal to the Sun's. Red dots indicate current semimajor axes of planets that will be engulfed, and tidally disrupted by their host stars. Blue circles indicate that the planet will avoid a common envelope phase with predicted final semimajor axes denoted by green dots. None of the systems shown will evolve to have circum-white dwarf planets within $\sim 6$ AU. This implies that in the absence of scattering events, first-generation planets around white dwarfs are unlikely to be found in short-period orbits.

Nevertheless, these systems represent a source of accretion onto the white dwarf as they will tidally disrupt when the differential gravitational force from the proto-white dwarf exceeds the self gravity of the planet. Figure 3 shows the orbital period where tidal disruption (shredding) occurs (yellow dash-dot line) as a function of companion mass. Companions in the magenta region can survive the CE phase and emerge in short-period orbits to white dwarfs. Known low-mass-short-period companions to white dwarfs are denoted by blue circles and black diamonds. Grey-scale and marker size indicate white dwarf mass.

Note that for planetary masses, the allowable parameter space where planets survive the CE is very limited. This implies that most planets will tidally disrupt during the CE. Such disruption events result in initially geometrically-thick-hyper-critical accretion disks within the common envelope that are cold and dense compared to the hot stellar interior. These disks are prone to powerful outflows and may produce highly-magnetized white dwarfs via a dynamo (Nordhaus et al. 2011). 


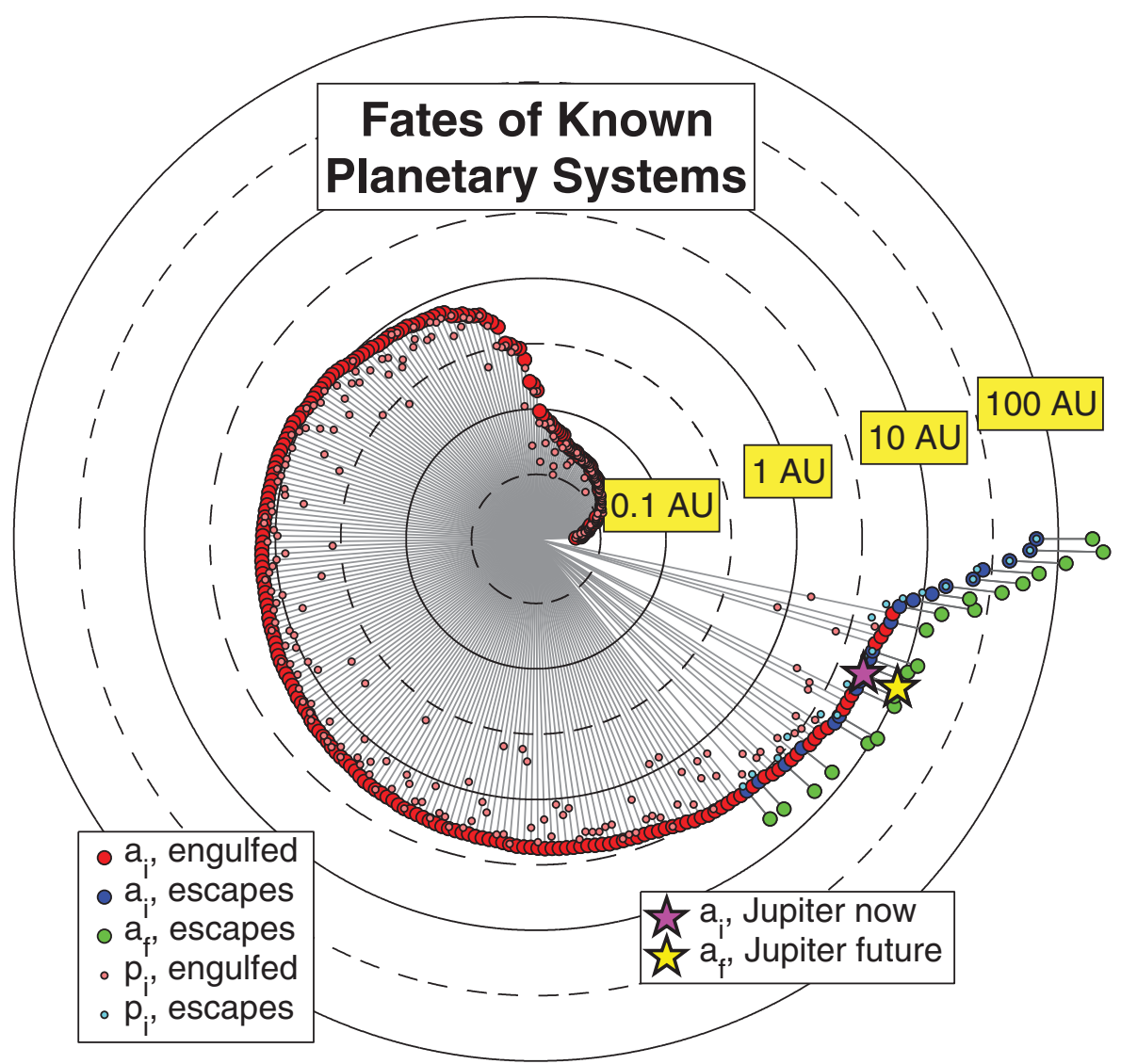

Figure 2. The fates of known planetary systems (Nordhaus \& Spiegel 2013). Red and blue dots indicate current semimajor axes for exoplanets with masses at least Jupiter's and host-star masses greater than or equal to the Sun's. A red dot indicates that the planet will be engulfed while a blue dot indicates that the planet will escape engulfment; green dots indicate final circum-WD separations. Small light-red (light-blue) dots indicate current periastra of planets that will be (avoid being) engulfed. Current and final orbital semimajor axes of Jupiter are shown with magneta and yellow stars, respectively. Among the currently known planetary systems, none will evolve to have circum-WD jovian planets within $~ 6.4 \mathrm{AU}$.

\section{Common Envelope Simulations}

The common envelope phase occurs on dynamical timescales, is highly-non spherical and thus requires three-dimensional simulations with sufficient physical fidelity to properly model. In the last several years, grid-based attempts to simulate CE phases have been performed. Grid-based codes have an advantage over smooth-particle-hydrodynamics as they capture shocks (the companion's motion in the CE is supersonic) and utilize dynamical resolution if adaptive-mesh techniques are employed. Recent hydrodynamic gridbased simulations have been carried out with the ENZO, FLASH, and AREPO codes and have focused on near-equal mass binaries (see Table 3; Iaconi et al. 2016, Ricker \& Taam 2012, Ohlmann et al. 2016). In these studies, the orbital energy available to unbind the $\mathrm{CE}$ should be sufficient even if radiative losses were severe. Under these conditions, none fully ejects the $\mathrm{CE}$ despite observing the dynamical inspiral phase. Additionally, all three predict final separations that are larger than those for these systems' observed post-CE counterparts. The authors conclude that processes on thermal timescales, unresolved 


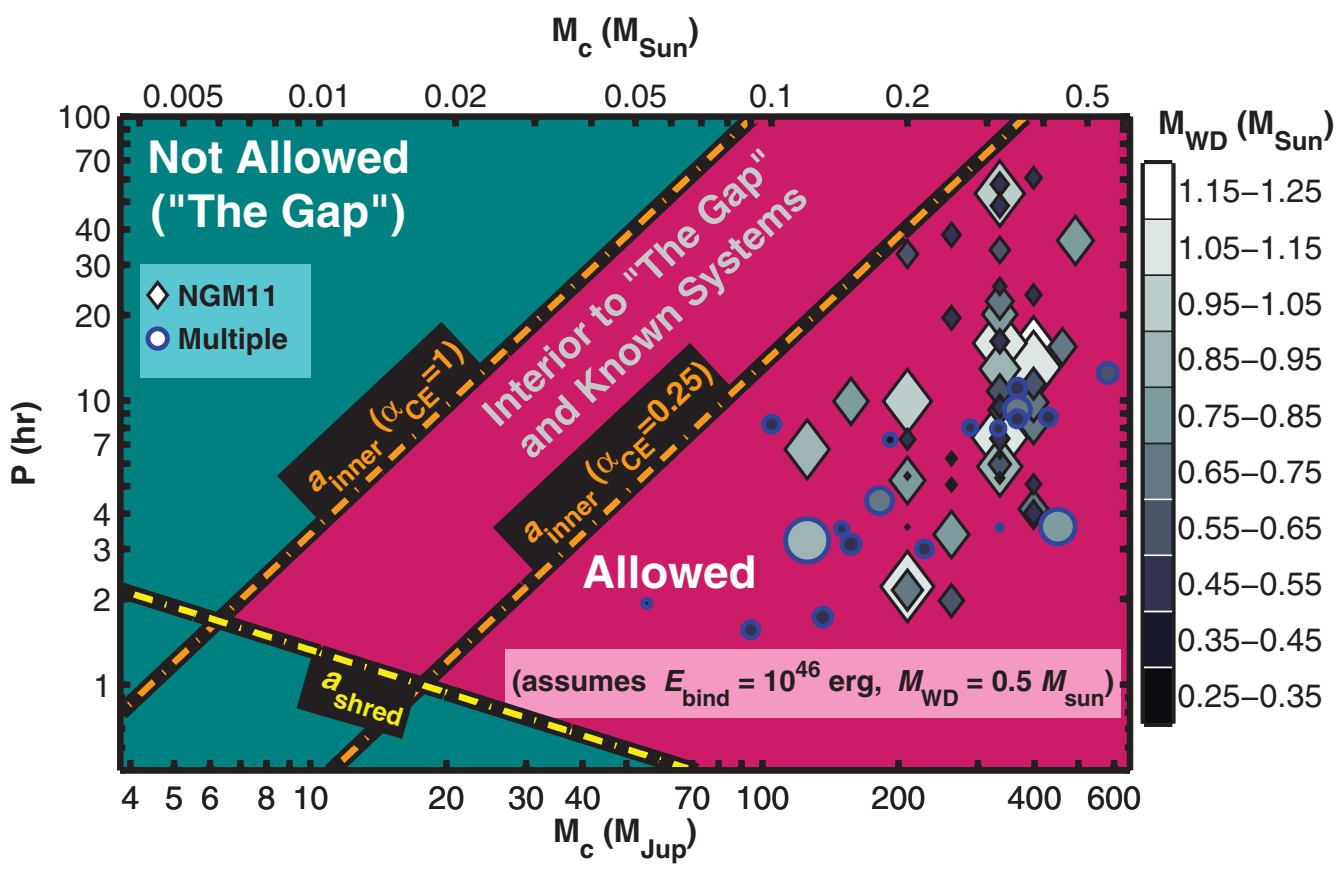

Figure 3. Known low-mass-short-period companions to white dwarfs (blue circles and black diamonds) compared to theoretical outcomes of CE evolution (Nordhaus \& Spiegel 2013). Objects in the magenta region can survive the CE phase and emerge as short-period companions to WDs. The $a_{\text {inner }}$ boundaries are determined by assuming that $\alpha_{\mathrm{CE}}$ of the liberated orbital energy during inspiral is used to unbind the CE. The $a_{\text {shred }}$ boundary marks the location where companions tidally disrupt. Greyscale and marker size indicate white dwarf mass.

Table 1. Overview of current grid-based common envelope simulations.

\begin{tabular}{|c|c|c|c|c|}
\hline Code & Primary $\left[M_{\odot}\right]$ & Secondary $\left[M_{\odot}\right]$ & Resolution $^{*}[\mathrm{~cm}]$ & Domain size $[\mathrm{cm}]$ \\
\hline $\mathrm{FLASH}^{1}(\mathrm{AMR})$ & 1.05 & 0.6 & $2 \times 10^{10}$ & $4 \times 10^{13}$ \\
\hline $\mathrm{ENZO}^{2}(\mathrm{AMR})$ & 0.88 & 0.6 & $1.2 \times 10^{11}$ & $6 \times 10^{13}$ \\
\hline $\mathrm{AREPO}^{3}$ (moving mesh) & 1.98 & 0.99 & $7 \times 10^{8}$ & $3.3 \times 10^{14}$ \\
\hline
\end{tabular}

Notes:

* Refers to smallest resolution present in the simulation.

1 Ricker \& Taam 2012

${ }^{2}$ Iaconi et al. 2016

${ }^{3}$ Ohlmann et al. 2016

microphysics, or other missing physical effects must be required to successfully eject the CE and produce post-CE systems that match observations (Ohlmann et al. 2016). Despite the initial challenges, these simulations represent the first step toward credible simulations of common envelopes. Future work must incorporate additional effects that are expected to be meaningfully important. These include, but are not limited to, incorporating magnetic fields, fully resolving convection in the giant's envelope, and including general relativistic effects for compact-object companions. Future work must also simulate a range of mass ratios for common envelope systems with various primaries. 


\section{References}

Blackman, E. G. \& Lucchini, S. 2014, Mon. Not. R. Astron. Soc., 440, L16

Bujarrabal, V., Castro-Carrizo, A., Alcolea, J., \& Sánchez Contreras, C. 2001, Astron. Astrophys., 377,868

Chen, Z., Nordhaus, J., Frank, A., Blackman, E. G., \& Balick, B. 2016, MNRAS, 460, 4182

Fabrycky, D. \& Tremaine, S. 2007, ApJ, 669, 1298

Fujii, Y., Spiegel, D. S., Mroczkowski, T., et al. 2016, ApJ, 820, 122

Iaconi, R., Reichardt, T., Staff, J., et al. 2016, MNRAS, in press.

Nordhaus, J. \& Blackman, E. G. 2006, MNRAS, 370, 2004

Nordhaus, J., Blackman, E. G., \& Frank, A. 2007, MNRAS, 376, 599

Nordhaus, J., Spiegel, D. S., Ibgui, L., Goodman, J., \& Burrows, A. 2010, MNRAS, 408, 631

Nordhaus, J., Wellons, S., Spiegel, D. S., Metzger, B. D., \& Blackman, E. G. 2011, Proceedings of the National Academy of Science, 108, 3135

Nordhaus, J. \& Spiegel, D. S. 2013, MNRAS, 432, 500

Ohlmann, S. T., Röpke, F. K., Pakmor, R., Springel, V., \& Müller, E. 2016, MNRAS, 462, L121

Paczynski, B. 1976, Structure and Evolution of Close Binary Systems, 73, 75

Ricker, P. M. \& Taam, R. E. 2012, ApJ, 746, 74

\section{Discussion}

Guzman-RAmirez: Are there observational constraints that will indicate that a single star has experienced a CE?

NorDhaus: For RGB/AGB stars, some possible indication might be isotopic abundances from extra mixing processes induced by inspiral of perhaps enhanced surface rotation rates.

De MARCO: The Kozai-Lidov effect might be efficient, but only a very small combination of parameters could give you a compact binary in the middle of a PN that has avoided a common envelope phase.

NorDhAus: The exact population calculation has not been worked out, but there might be more of these objects than one may imagine. 